\title{
Illegitime Geschäfte in der „Coronakratie“ - ethische Perspektiven auf die Einflussnahme durch politische Entscheidungsträgerinnen und -träger
}

\author{
Maximilian Schiffers
}

Angenommen: 29. Juni 2021 / Online publiziert: 19. Juli 2021

(C) Der/die Autor(en) 2021

\section{Der Maskenskandal als Auslöser einer Transparenzdebatte}

Im Frühjahr des Jahres 2021 - zu Beginn der dritten Welle der Corona-Pandemie in Deutschland - diskutierte die Republik das Thema der Corona-Schutzmasken unter einem besonderen Vorzeichen. Bei der Vermittlung von dringend benötigten Schutzmasken an das Bundesgesundheitsministerium haben sich einige Abgeordnete persönlich bereichert. Es standen Vorwürfe illegitimer Einflussnahme, politischer Korruption, der Bestechlichkeit von Abgeordneten und Steuerhinterziehung im Raum. Die öffentliche Empörung und mediale Dramatisierung des Normbruches folgte den bekannten Phasen der Skandalisierung (Burkhardt 2015, Schicha 2021), in deren Verlauf immer neue Details und neue Verstrickungen ans Licht kamen. Im Mittelpunkt befanden sich eine Reihe von CDU/CSU-Abgeordneten im Bundestag und in Landtagen, die auf verschiedene Weisen lukrative Geschäfte aus der Coronapolitik der Pandemie schlagen konnten. In der politischen Debatte ordneten sich die Fälle in einen bereits bestehenden Resonanzraum ein. Denn vielfache Skandale und Affären, wie die Maskengeschäfte, die Aserbaidschan-Connection sowie als illegitim wahrgenommene Lobbying-Aktivitäten führten zu einer breiten Diskussion, welche moralischen und ethischen Anforderungen an Bundestagsabgeordnete gestellt werden sollen, die die rein rechtlichen Schranken flankieren. Da sich die problematischen Fälle vielfach in der juristischen Aufarbeitung befinden, lässt sich als vereinfachender Ausgangspunkt festhalten, dass sich die politikwissenschaftliche Einordnung maßgeblich im Bereich des politisch Illegitimen jedoch rechtlich Legalen bewegt. (vgl. Wolf 2021, S. 27; Graeff 2021, S. 16). Auch die politische Reaktion folgte prompt. So lässt sich schlüssig argumentieren, dass die zeitnahe

\footnotetext{
Maximilian Schiffers $(\bowtie)$

NRW School of Governance, Universität Duisburg-Essen, Lotharstr. 53, 47057 Duisburg,

Deutschland

E-Mail: maximilian.schiffers@uni-due.de
} 
Reihung der Skandale dazu beigetragen hat, die politischen Reibungen rund um das Thema der Transparenz- und Nachvollziehbarkeitsregeln so zu steigern, dass die Unionsparteien ihre langjährige Blockadehaltung aufgeben mussten und somit den Weg frei machten für die Einführung eines gesetzlichen Lobbyregisters.

Der Essay schließt an diese Frage an und eröffnet eine ethische Perspektive auf die Grenzen zwischen illegitimer Einflussnahme und den Grauzonen legaler Geschäfte in der „Coronakratie“ (Florack et al. 2021). Zunächst folgt ein Überblick über die zentralen Transparenzmaßnahmen und über die strukturellen Lücken bisheriger Regelungen. Anschließend werden ausgewählte Beispiele illegitimer Einflussnahme anhand von drei Formen der Ethik - Konsequentialismus, Deontologie, Tugendethik - erklärt. Im Fazit wird begründet, warum die bisherigen Regelungen zur Grenzziehung zu ungenau sind und somit zum Problem des Vertrauensverlusts in die Demokratie beitragen.

\section{Bisherige Regelungen und Lücken}

Politische Skandale, die mutmaßlich in vielen Teilen nach dem Buchstaben des Gesetzes legal sind, deuten auf das strukturelle Problem eines zu niedrigen Regelungsniveaus der Transparenz- und Nachvollziehbarkeitspolitik hin. Denn Treiber der Debatte sind nicht nur die Skandale - die aufgrund der lange bekannten Schwachstellen gewissermaßen mit Ansage kamen - sondern auch die internationalen Verpflichtungen (Crepaz 2017; Schiffers 2018). Die Staatengruppe gegen Korruption im Europarat, GRECO, kritisiert seit 2015 regelmäßig, dass Deutschland die internationalen Standards für Parteienfinanzierung, Interessenkonflikte und Unternehmensbeteiligungen von Abgeordneten sowie Lobbying-Regulierung wiederholt nicht einhalte. Das Gremium hatte bereits 2019 ein Nichteinhaltungsverfahren gegen Deutschland eingeleitet, im Mai diesen Jahres folgte eine weitere Rüge. Hierbei ging es konkret um die fehlende Prävention von Abgeordnetenbestechung und Mängel bei Kontrolle und Durchsetzung von Regeln (Deutsche Welle 2019; GRECO 2020; Der Tagesspiegel 2021).

Systematisch gesprochen liegen die Ziele von Transparenz- und Nachvollziehbarkeitsregeln darin, die Legitimität des demokratischen Staates in seiner Input-, Throughput- und Output-Dimension (vgl. Schmidt 2013) zu sichern und das Vertrauen der Bürgerinnen und Bürger in politische Institutionen zu stärken. Damit greift das Öffentlichkeitsprinzip in der Demokratie: Information und Transparenz über politische Aktivitäten müssen zugänglich sein. Erreicht wird dies durch barrierefrei öffentlich gemachte Informationen der Verwaltung sowie durch die Nachvollziehbarkeit von Geldflüssen, Mittelverwendung, politischer Einflussnahme und von Entscheidungskriterien. Politische Maßnahmen und Steuerungsinstrumente zielen auf das Zusammenspiel von Transparenz und Gewaltenteilung, Strafbewehrung und interner Kontrolle, sowie auf Prävention durch Organisationskultur, ökonomischer Anreize und Wissensvermittlung (Wolf 2014, S. 68-70; 2021, S. 26; Fütterer 2018, S. 34-35, 43-44; Mugellini 2020, S. 7-8).

Die verschiedenen Instrumente im Werkzeugkasten der Transparenz- und Nachvollziehbarkeitsregeln sind in Deutschland auf eine Reihe von gesetzlichen Grund- 
lagen aufgeteilt. Prominenteste Reaktion war der Beschluss eines gesetzlich verpflichteten Lobbyregisters im März 2021, dem eine langjährige Auseinandersetzung zwischen den Koalitionsparteien vorausging. Auf öffentlichen Druck - auch von Seiten von Lobbyakteuren, Verbänden und NGOs, die das Lobbyregister mehrheitlich einforderten - gaben CDU/CSU ihre Blockadehaltung auf. Die Einschätzung der Expertinnen und Experten aus der Praxis und der Wissenschaft fiel gemischt aus. Die angestrebte Lobbyregulierung liege im internationalen Vergleich lediglich im Mittelfeld, zentrale Registerkategorien, wie Lobbying-Ziel, -instrumente und Kontaktoffenlegung fehlten (Deutsche Welle 2021). Als weitere Folge der sogenannten Maskenaffäre legten die Regierungs- und Oppositionsfraktionen im Bundestag zahlreiche Vorschläge für Transparenzregeln vor, die u. a. das Abgeordnetengesetz betreffen. Der Gesetzentwurf ,zur Verbesserung der Transparenzregeln für die Mitglieder des Deutschen Bundestages“ wurde im Juni 2021 beschlossen (Deutscher Bundestag 2021; zur kritischen Würdigung der Inhalte siehe Abgeordnetenwatch 2021). Gleichzeitig veröffentlichte das Bundeskabinett den Verhaltenskodex für Interessenvertreterinnen und -vertreter, der im Rahmen des Lobbyregistergesetzes vorgesehen ist (BMI 2021) (Tab. 1).

Strukturell wird die politische Debatte geprägt von einem dissonanten Verhältnis der Unionsparteien zu Transparenz. Die Partei konnte über viele Jahre eine Blockade gegen verschiedene Transparenzmaßnahmen aufrechterhalten. Darunter fielen zum einem aktuell diskutierte und beschlossene Initiativen, wie das Lobbyregister, Regeln für Abgeordnetenbestechung und Nebentätigkeiten, zum anderen das politische Ziel der Deregulation und des Bürokratieabbaus. Geradezu entgegengesetzt ist das

Tab. 1 Werkzeugkasten der Transparenzmaßnahmen und aktuelle Reformen. (Eigene Darstellung)

Zentrale Instrumente im Werkzeugkasten der Transparenzmaßnahmen

Parteiengesetz (1967, 1994, 2020) mit Parteifinanzierung, das Parteispenden reguliert und kontrolliert (seit 2002 ist Herkunftsverschleierung eine Straftat)

Abgeordnetengesetz (1977, 1996, 2020, 2021), u. a. mit Regelungen zu Nebentätigkeiten und Anzeigepflichten für Einkünfte

Antikorruptionskonvention der UN von 2005, die Abgeordnetenbestechung unter Strafe stellen verbunden mit dem Strafgesetzbuch ( $\$ 108$ e StGB) Bestechlichkeit und Bestechung von Mandatsträgern (1994/2014)

Informationsfreiheitsgesetz (seit 2006): Zugang zu amtlichen Informationen

Karenzzeiten-Gesetz (seit 2015) zum Wechsel aus Politik zu Wirtschaft von Ministerinnen und Staatssekretärinnen

Geschäftsordnung von Bundestag (GOBT) mit Verhaltensregeln für Mitglieder des Deutschen Bundestages sowie Gemeinsame Geschäftsordnung der Bundesministerien (GGO)

Lobbyregister (ab 2022) in gesetzlich verpflichtender Variante auf mittlerem Regulierungsniveau im internationalen Vergleich

Verhaltenskodex ( $a b$ 2022) für Interessenvertreterinnen und -vertreter im Rahmen des Lobbyregistergesetzes
Reform zu niedrigeren Grenzen der Veröffentlichungspflicht für Parteispenden blockiert

Mit Reform zum Verbot von Mandatslobbying im Juni 2021 (Drucksache 19/28784)

Reformvorschlag 2021 blockiert

Reform zur Einführung eines Verhaltenskodex wird diskutiert (Drucksache 19/30885)

Beschlossen im März 2021 (LobbyRG vom 16. April 2021)

Kabinettsbeschluss vom 16 . Juni 2021 (BMI 2021) 
Transparenzverständnis in anderen Politikfeldern. Im digitalen Raum stehen Teile der Partei beispielsweise für Klarnamenpflicht, Telekommunikationsüberwachung und den sogenannten Staatstrojaner, im Sozialbereich für Offenlegungspflichten für Hartz IV, beim Elterngeld oder für Corona-Hilfen. Gerade diese Dissonanz führte in der aktuellen Debatte zu diesem hohen Maß an Kritik (zur Übersicht der Kritik, Zeit Online 2021; Tagesschau.de 2021b).

\section{Ethische Perspektiven auf zentrale Aspekte der Problemfälle}

In der politisch-philosophischen Auseinandersetzung hat sich eine Dreiteilung der zentralen ethischen Perspektiven etabliert, die vielfach als ,three forms of political ethics“ (McDonald 1978) oder ,three methods of ethics“ (Baron et al. 1997; vgl. Pieper 2017; Bratu und Nida-Rümelin 2013) charakterisiert werden. Diese vereinfachenden, idealtypischen Perspektiven - Deontologie, Tugendethik und Konsequentialismus - legen verschiedene Maßstäbe an, auf deren Grundlage normative Bewertungen erfolgen können. Die problematischen Fälle ${ }^{1}$ haben dazu beigetragen, dass auch weitere, anders gelagerte politische Vorhaben äußerst kritisch gesehen werden. So lässt sich verstehen, warum die Kritik an der Maskenbeschaffung durch das Bundesgesundheitsministerium im Zeitraum ab Beginn der Corona-Pandemie im Frühjahr 2020, von der neben den beteiligten Politikern insbesondere Apotheken finanziell profitierten, nun heftig vorgebracht wird. Denn vielfach entsteht der Eindruck, dass es sich um dieselbe Kerbe der illegitimen Einflussnahme handeln, in die alle Fälle einschlagen. Im Folgenden werden verschiedene Aspekte der Fälle herausgearbeitet, die Bezug zu den Transparenzregeln haben und aus jeweils einer Perspektive beleuchtet werden.

Die deontologische bzw. Pflichtethik sieht bestimmte handlungsleitende Pflichten und Rechte vor. Diese müssen erfüllt sein, um Handlungen als geboten anzusehen. Gleichsam verbieten sie Handlungen, die diese Pflichten und Rechten verletzen würden. Zentrale Strömungen in dieser Perspektive berufen sich auf Kant als ,archetypical deontologist“" und Benthan als ,,classical“ utilitarian“ (McDonald 1978, S. 7). In dieser ,deontischen Logik“ ergeben sich eine Reihe von Handlungsalternativen, die erlaubt, geboten oder verboten sein können (Pieper 2017, S. 175.) - und zwar unabhängig von ihren Konsequenzen (Bratu und Nida-Rümelin 2013, S. 5). Für Abgeordnete lässt sich in diesem Sinne von einer Pflicht zu intrinsisch korrektem Verhalten sprechen. Denn sie haben den eigenen Anspruch als Vorbild, welcher Politikerinnen durch ihre gesellschaftlich und medial exponierte Stellung zugeschrieben wird. Diesem verpflichtenden Anspruch müssen die Mandatsträgerinnen jedoch aktiv gerecht werden.

Dazu passende Aspekte lassen sich in den Fällen der Bundestagsabgeordneten Georg Nüßlein (ehemals CSU), Nikolas Löbel (ehemals CDU) und Mark Hauptmann (ehemals CDU) finden. Nüßlein und Löbel erhielten für ihre Vermittlung zwi-

\footnotetext{
1 Bei den Fällen handelt es sich in dieser Debatte um Politikerinnen und Politiker der CDU/CSU. Expertinnen und Experten betonen, dass sie ähnlich gelagerte Fälle - wenn auch weniger häufig - auch bei anderen Parteien finden lassen (zur Übersicht siehe Zeit Online 2021).
} 
schen Firmen und Ministerium zu marktunüblich überhöhten Preisen Provisionen im sechsstelligen Bereich. Im Fall Nüßlein lief die Zahlung über ein Firmengeflecht und eine Liechtensteiner Bank, worauf hin die Finanzbehörden und die Generalstaatsanwaltschaft tätig wurde. Im Fall Hauptmann erhielt sein Kreisverband eine Parteispende einer vermittelten Firma. Alle drei Politiker traten als Folge aus ihrer Partei und Fraktion aus, Hauptmann legte zudem sein Mandat nieder. Pflichtethisch relevant ist insbesondere der individuelle Vorteil, den sich die drei Abgeordneten auf Grundlage ihrer exponierten und vertrauensvollen Stellung sicherten, indem sie den Firmen privilegierten Zugang ermöglichten (,access corruption“, Ron und Singer 2020, S. 53). Sie verstießen gegen die normative Pflicht, keinen Schaden für die Steuerzahlenden durch persönliche Verfehlung zu verursachen. Denn ohne konkretes Gesicht bleiben die Steuerzahlenden ein abstraktes Opfer (zur Problematisierung von Korruption als sogenanntes „opferloses Delikt“ siehe Wolf 2014, S. 30). Der hohe, normativ verpflichtende Anspruch, der sich in der politischen Debatte zeigte, leidet allerdings schon dadurch, dass sie sich dem Vorwurf eines Interessenkonflikts aussetzen mussten.

Die Tugendethik betont den Charakter einer Person, in welchem spezifische tugendhafte Fähigkeiten in besonderem, gutem Maß ausgeprägt sein sollen. Sie stellt damit ein „Gegenmodell zu handlungsorientierten ethischen Positionen“ dar (Bratu und Nida-Rümelin 2013). Diese Perspektive geht zurück auf Aristoteles und die Charaktertugenden, wie Besonnenheit, Tapferkeit und Gerechtigkeit, um das Ideal des guten Lebens zu erreichen (Pieper 2017, S. 243-244). Zeitgenössische Ansätze unterscheiden, welche Auswahl und Ausprägung von Tugenden relevant sind. Für politische Entscheidungsträgerinnen wird demnach das richtige, moralische Handeln als Charaktermerkmal zum Ideal.

Dazu passende Aspekte liefert der Fall des bayerischen Landtagsabgeordneten und ehemaligen Justizministers Alfred Sauter (ehemals CSU). Kritisiert wurde seine parallele Tätigkeit als Abgeordneter und als Anwalt, bei der er u.a. am Vertrag zwischen Ministerium und eines Maskenlieferanten mitwirkte. Tugendethisch relevant ist insbesondere die systematische Netzwerkbildung Sauters mit Unternehmen und Personen aus der Wirtschaft, um Parteispenden einzuwerben. Im Vordergrund stand nicht (ausschließlich) der individuelle Nutzen, sondern ein - zumindest speziell verstandenes - Pflicht- und Loyalitätsgefühl gegenüber der Partei (zu Korruption und Gruppenloyalität siehe Graeff 2021, S. 19-20). Die Charaktertugend Loyalität muss hier die Abwägung treffen zwischen Partei und Staat. Vergleichbare problematische Netzwerke mit inhaltlichen Überschneidungen finden sich etwa bei Forderungen von Verbänden, die den Parteien nahestehen, wie Eigentümer- und Vermieterverbänden bei der Mietpreisbremse, Apothekenverbände bei der Maskenverteilung (zur Übersicht, Die Anstalt 2021). Ebenfalls passende Aspekte liefern der Verhaltenskodex der CSU, der an hohe Ideale appelliert sowie der sich noch in Arbeit befindliche Verhaltenskodex der Unionsbundestagsfraktion (Tagesschau.de 2021a, b). Das tugendethische Argumentationsmuster verweist hier auf das Fehlverhalten einzelner schwarzer Schafe, die die freiwillig gesetzten Ideale verletzen (analog zum Verhaltenskodex im Lobbyregister). So lässt sich zeigen, dass Lobbyregister zur wechselseitigen Kontrolle der Lobbyakteure untereinander genutzt werden (Crepaz 2020). 
Abseits der ethischen Bewertung muss die Wirkung von Selbstverpflichtungen ohne Sanktionsmöglichkeiten jedoch kritisch geprüft werden.

Die konsequentialistische Ethik bewertet danach, welche Folgen eine Handlung hat und wie die bestmöglichen Konsequenzen für alle Beteiligten angestrebt werden können. Damit schließt die Perspektive Strömungen des Utilitarismus und der Nutzenmaximierung bzw. der Abwehr vor Schaden (Pieper 2017, S. 164-166). Zentrale Prinzipien sind die Klärung, welches die relevanten intrinsischen Werte sind, wie diese Werte durch eine Handlung aggregiert werden und die Auswahl, welche Handlungsalternative die höchstbewerteten Werte am besten realisiert (Bratu und Nida-Rümelin 2013, S. 4). Dies schließt an die politikwissenschaftliche Debatte über Output-Legitimation an. Die Qualität politischer Ergebnisse von Institutionen werden daran gemessen, dass sie besonders wirksame Folgen für die Bevölkerung haben. Als Legitimationsmechanismus steht das Prinzip „,for the people“ gegenüber Partizipation (,by the people“) und Repräsentation (,of the people“) im Vordergrund (siehe Schmidt 2013).

Dazu passende Aspekte liegen in der Gesamtschau der Fälle der ausschnitthaften Jahre 2019-21 mit der Gegenüberstellung von persönlicher Besserstellung bzw. Bereicherung gegen den politischen Schaden durch verlorenes politisches Vertrauen in Abgeordnete, die Partei und in Politik als Ganzes (vgl. Oberle 2021, S. 10-11; Crouch 2021, S. 85-86). Dieser Schaden teilt sich auf in die persönlichen Konsequenzen für die politischen Karrieren der Beteiligten sowie in die Wirkung für die Partei im Superwahljahr. Die lange Geschichte von Korruptionsskandalen und die mutmaßlich strukturellen Probleme der Partei legen die Assoziation mit Betrug und illegitimer Einflussnahme nahe (ausführlich im Faktencheck, Die Anstalt 2021). Mildernd lässt sich argumentieren, dass die Notlage der Pandemie schnelles Handeln erforderte. Die Vermittlungstätigkeiten wären demnach ,Speedmoney“ (Graeff 2021, S. 17) als Ersatzstrategie, wenn die Prozesse der Interessenvermittlung und des Lobbyings zu lange dauern (Goldberg 2018). Zynisch ließe sich formulieren, dass ,kein Schaden ohne Nutzen“ ist, wenn man die durchbrochene Blockadehaltung gegen Transparenzmaßnahmen als Konsequenz mit einbezieht. Allerdings ist die Gefahr des politischen Vertrauensverlusts in Institutionen und Demokratie sehr dramatisch, weil Vertrauen ein zentraler Faktor für das Funktionieren einer Demokratie ist. Als zentrale Währung auf dem Wählermarkt genügt auch hier bereits der Anschein von Käuflichkeit, um dem Ansehen der Politik zu schaden.

\section{Grenze für illegitimes und trotzdem legales Verhalten ist zu unscharf und zu lasch.}

Verfehlte Anspruchsideale aus Perspektive der Pflichtethik, falsch verstandene Loyalität gegenüber der eigenen Partei statt der Bevölkerung aus Perspektive der Tugendethik sowie nachhallender Schaden für das Vertrauen in Politik und demokratische Institutionen aus Perspektive des Konsequenzialismus: Die ethisch-moralischen Anforderungen an politische Entscheidungsträgerinnen und -träger sind insbesondere abseits der rein rechtlichen Schranken von zentraler Bedeutung. Denn die bisherigen Transparenzmaßnahmen haben noch immer Schwachstellen und Lücken. 
Der Essay thematisierte drei Perspektiven auf die Grauzonen illegitimer, doch mutmaßlich legaler Geschäfte von Abgeordneten in der „Coronakratie“. An den skizzierten Werkzeugkasten der Transparenzmaßnahmen setzen bereits aktuelle Reformen, wie Lobbyregister und Abgeordnetengesetz an. Allerdings genügt es nicht, legalistisch auf den Buchstaben des Gesetzes oder auf das Fehlverhalten einzelner zu verweisen. In der Zusammenschau unterstreicht die ethische Betrachtung vielmehr, dass politische Nachvollziehbarkeit und Transparenz durch formale und normative Pflichten, Tugendideale und Output-Qualitäten materiell ausgefüllt werden muss. Sie konkretisiert damit die vielfach diskutierte normative wie analytische Herausforderung des demokratischen Vertrauens.

Denn wie diese Grenze zwischen legalen und illegitimen Einflussformen von Bürgerinnen und Bürgern wahrgenommen wird, ist im demokratischen Miteinander zentral. Die juristischen Feinheiten und die konkreten Prozessabläufe sind vielfach zu knifflig, um sie ohne vertiefte Lektüre verstehen zu können. Was bleibt ist der schale Geschmack, dass sich politische Entscheidungsträgerinnen und -träger inmitten einer Ausnahmesituation durch illegitime Geschäfte persönlich bereichert haben und nur zögerlich dafür zur (politischen) Rechenschaft gezogen wurden. Das trägt in besonderem Maße zur Politikverdrossenheit bei (Oberle 2021, S. 10-11; vgl. Crouch 2021, S. 76, 86). In der Zusammenschau unterstreichen die drei ethischen Perspektiven genau diese normative wie analytische Herausforderung des demokratischen Vertrauens. Die Problemfälle des Jahres 2021 erzeugten das Momentum, um verzögerte und teils blockierte Reforminitiativen anzupacken. Das Thema der politischen Nachvollziehbarkeit durch Transparenz und Rechenschaft bleibt so lange Maßstab im Superwahljahr und auf der Agenda für die nächste Legislaturperiode, bis das erschütterte Vertrauen der Menschen in ihre Abgeordneten und in die Politik zurückerarbeitet wird. Denn bereits der Anschein von Interessenkonflikten und Käuflichkeit schadet dem Ansehen der ganzen Politik und dem normativen Anspruch an Mandatsträgerinnen und -träger in modernen Demokratien.

Funding Open Access funding enabled and organized by Projekt DEAL.

Open Access Dieser Artikel wird unter der Creative Commons Namensnennung 4.0 International Lizenz veröffentlicht, welche die Nutzung, Vervielfältigung, Bearbeitung, Verbreitung und Wiedergabe in jeglichem Medium und Format erlaubt, sofern Sie den/die ursprünglichen Autor(en) und die Quelle ordnungsgemäß nennen, einen Link zur Creative Commons Lizenz beifügen und angeben, ob Änderungen vorgenommen wurden.

Die in diesem Artikel enthaltenen Bilder und sonstiges Drittmaterial unterliegen ebenfalls der genannten Creative Commons Lizenz, sofern sich aus der Abbildungslegende nichts anderes ergibt. Sofern das betreffende Material nicht unter der genannten Creative Commons Lizenz steht und die betreffende Handlung nicht nach gesetzlichen Vorschriften erlaubt ist, ist für die oben aufgeführten Weiterverwendungen des Materials die Einwilligung des jeweiligen Rechteinhabers einzuholen.

Weitere Details zur Lizenz entnehmen Sie bitte der Lizenzinformation auf http://creativecommons.org/ licenses/by/4.0/deed.de. 


\section{Literatur}

Abgeordnetenwatch. 2021. Kehrtwende bei Verhaltensregeln. Das neue Abgeordnetengesetz: Stärkere Regeln, schwache Kontrolle. Vom 11.06.2021. https://www.abgeordnetenwatch.de/blog/lobbyismus/ das-neue-abgeordnetengesetz-staerkere-regeln-schwache-kontrolle. Zugegriffen: 23. Juni 2021.

Baron, Marcia, Philip Pettit, und Michael Slote. 1997. Three methods of ethics. Cambridge und London: Blackwell.

BMI [Bundesministerium des Inneren, für Bau und Heimat]. 2021. Bundeskabinett beschließt Verhaltenskodex für Interessenvertreterinnen und Interessenvertreter. Vom 16.06.2021. https://www.bmi.bund. de/SharedDocs/kurzmeldungen/DE/2021/06/verhaltenskodex-lobbyregistergesetz.html. Zugegriffen: 23. Juni 2021.

Bratu, Christine, und Julian Nida-Rümelin. 2013. Argumentationslinien der praktischen Philosophie. Bundezentrale für politische Bildung. https://www.bpb.de/gesellschaft/umwelt/bioethik/162732/ argumentationslinien-der-praktischen-philosophie. Zugegriffen: 23. Juni 2021.

Burkhardt, Steffen. 2015. Medienskandale. Zur moralischen Sprengkraft öffentlicher Diskurse, 2. Aufl., Köln: Herbert von Halem.

Crepaz, Michele. 2017. Why do we have lobbying rules? Investigating the introduction of lobbying laws in EU and OECD member states. Interest Groups \& Advocacy 6:231-252.

Crepaz, Michele. 2020. To inform, strategise, collaborate, or compete: What use do lobbyists make of lobby registers? European Political Science Review 12(3):347-369.

Crouch, Colin. 2021. Postdemokratischer Kapitalismus. Zum Zusammenhang von Korruption und Ungleichheit. Blätter für deutsche und internationale Politik 2021(4):76-86.

Der Tagesspiegel (online). 2021. Neue Rüge für Deutschland: Bundestag tut zu wenig gegen Abgeordnetenbestechung. Vom 10.05.2021. https://www.tagesspiegel.de/politik/neue-ruege-fuer-deutschlandbundestag-tut-zu-wenig-gegen-abgeordnetenbestechung/27175430.html. Zugegriffen: 23. Juni 2021.

Deutsche Welle. 2019. Europarat fordert Regeln gegen Korruption in Deutschland. Vom 12.08.2019. https://p.dw.com/p/3Nm9M. Zugegriffen: 23. Juni 2021.

Deutsche Welle. 2021. Deutschland bekommt ein Lobbyregister. Vom 06.03.2021. https://p.dw.com/p/ 3qFZd. Zugegriffen: 23. Juni 2021.

Deutscher Bundestag. 2021. Erweiterte Transparenzregeln für Abgeordnete beschlossen. Vom 11.06.2021. https://dbtg.tv/fvid/7526786. Zugegriffen: 23. Juni 2021.

Die Anstalt. 2021. Der Faktencheck zur Sendung vom 04. Mai 2021. Vom 05.05.2021. https://www.zdf. de/comedy/die-anstalt/fakten-im-check-der-anstalt-118.html. Zugegriffen: 23. Juni 2021.

Florack, Martin, Karl-Rudolf Korte, und Julia Schwanholz (Hrsg.). 2021. Coronakratie: Demokratisches Regieren in Ausnahmezeiten. Frankfurt am Main: Campus.

Fütterer, Sabine. 2018. Korruption und Antikorruption in der Wissensvermittlung - Unschärfen und Abhängigkeiten. In Korruptionsbekämpfung vermitteln, Hrsg. Sebastian Wolf, Peter Graeff, 25-47. Wiesbaden: Springer VS.

Goldberg, Felix. 2018. Corruption and lobbying: conceptual differentiation and gray areas. Crime, Law and Social Change 70:197-215.

Graeff, Peter. 2021. Grauzonen moderner Korruption. Aus Politik und Zeitgeschichte 71(19/20):16-20.

GRECO Staatengruppe gegen Korruption des Europarates. 2020. Evaluierungsbericht Deutschland; Stand 15.10.2020. GrecoEval5Rep(2019)6

McDonald, Lee C. 1978. Three forms of political ethics. The Western Political Quarterly 31(1):7-18.

Mugellini, Giulia. 2020. Corruption. In The Palgrave encyclopedia of interest groups, lobbying and public affairs, Hrsg. Phil Harris, Alberto Bitonti, Craig S. Fleisher, und Anne Skorkjær Binderkrantz. Cham: Palgrave Macmillan.

Oberle, Monika. 2021. Korruption als Gegenstand der politischen Bildung. Aus Politik und Zeitgeschichte 71(19/20):16-20.

Pieper, Annemarie. 2017. Einführung in die Ethik, 7. Aufl., Stuttgart: utb.

Ron, Amit, und Abraham A. Singer. 2020. Democracy, corruption, and the ethics of business lobbying. Interest Groups \& Advocacy 9:38-56.

Schicha, Christian. 2021. Politikerskandale in der Medienberichterstattung. In Handbuch Regierungsforschung, Hrsg. Karl-Rudolf Korte, Martin Florack. Wiesbaden: Springer VS.

Schiffers, Maximilian. 2018. Schritte und Rückschritte der Debatte über gesetzliche Lobbyregulierung. Regierungsforschung.de. Das wissenschaftliche Online-Magazin der NRW School of Gov- 
ernance. https://regierungsforschung.de/schritte-und-rueckschritte-der-debatte-ueber-gesetzlichelobbyregulierung/. Zugegriffen: 23. Juni 2021.

Schmidt, Vivien A. 2013. Democracy and legitimacy in the European Union revisited: input, output and "throughput". Political Studies 61:2-22.

Tagesschau.de. 2021a. Verhaltenskodex der CSU. Strenge Regeln, warme Worte? Vom 12.03.2021. https:// www.tagesschau.de/investigativ/wdr/nuesslein-csu-verhaltenskodex-101.html. Zugegriffen: 23. Juni 2021.

Tagesschau.de. 2021b. Maskenaffäre in der Union. Wie steht es um die Konsequenzen? Vom 04.06.2021. https://www.tagesschau.de/inland/maskenaffaere-konsequenzen-cdu-korruption-101.html. Zugegriffen: 23. Juni 2021.

Wolf, Sebastian. 2014. Korruption, Antikorruptionspolitik und öffentliche Verwaltung. Einführung und europapolitische Bezüge. Wiesbaden: Springer VS.

Wolf, Sebastian. 2021. Korruption und Antikorruption in Politik und Verwaltung. Aus Politik und Zeitgeschichte 71(19/20):21-27.

Zeit Online. 2021. Maskenaffäre: Sind CDU und CSU besonders korruptionsanfällig? Vom 09.03.2021. https://www.zeit.de/politik/deutschland/2021-03/maskenaffaere-union-korruption-bundestagswahlgeorg-nuesslein-nikolas-loebel/komplettansicht. Zugegriffen: 23. Juni 2021. 